\title{
Optical Nonlinearities at the Interface between Glass and Liquid Crystal $\uparrow$
}

\author{
PEDRO MENÉNDEZ-VALDÉS and JOSÉ A. MARTIN-PEREDA \\ Universidad Politécnica de Madrid, Escuela Técnica Superior de Ingenieros de \\ Telecomunicación, 28040 Madrid, Spain
}

(Received August 8, 1986; in final form February 27, 1987)

In this paper, the optical behavior of a nonlinear interface is studied. The nonlinear medium has been a nematic liquid crystal, namely MBBA, and the nonlinear one, glasses of different types (F-10 and F-2) depending on the experimental needs. The anchoring forces at the boundary have been found to inhibit the action of the evanescent field in the case of total internal reflection. Most of observed nonlinearities are due to thermal effects. As a consequence, liquid crystals do not seem to be good candidates for total internal reflection optical bistability.

Keywords: nematic, nonlinear optics, bistability, interface, total internal reflection

\section{INTRODUCTION}

The optical behavior of the interface between a linear and a nonlinear material constitutes a very interesting subject in the field of nonlinear optics. Its fundamental applications are Optical Bistability (OB) and Nonlinear Optical Waveguides. ${ }^{1-6}$ The optical bistable behavior of nonlinear interfaces was studied by Kaplan in early works. ${ }^{7}$ Smith et al. ${ }^{8}$ developed interesting experimental work to nonlinear interfaces with different nonlinear materials in the search for OB. Some time ago, nematic liquid crystals have been introduced as nonlinear material for such interfaces. The first studies by $\mathrm{Khoo}^{9}$ have shown a switching action mainly due to a thermal effect. Some other studies have been carried out using non-aligned nematic liquid crystals, in

Paper presented at the 11th International Liquid Crystal Conference, Berkeley, CA, 30 June-4 July, 1986. 
single interfaces by us, ${ }^{10}$ and in optical waveguides by other authors. ${ }^{11,12}$ In the case of optical bistability, the aim of the experimentation is, in most cases, to obtain a switch from Total Internal Reflection (TIR) to transmission. This switching has been shown by Kaplan to present a bistable hysteresis.

In nematic liquid crystals, nonlinearities are due to two different phenomena: optically induced molecular reorientation, and laser heating giving rise to thermal indexing. ${ }^{13,14}$ Let us associate the nonlinearity with the sign of the third order term in the susceptibility. Then, the molecular reorientation induces a "positive" nonlinearity both in nematic and isotropic phases. The laser heating induces a "negative" but very weak nonlinearity in the isotropic phase of a nematic compound (IP), while a positive one on the ordinary index of the nematic phase (NP). Therefore, laser heating could mask a possible reorientation effect in the nematic phase at the interface ${ }^{9,15}$ or oppose it in isotropic phase.

The aim of the experiments described in this paper is to establish the role of each one of the above effects. The possibility for reorientation to be induced by the evanescent field in spite of the strong anchoring forces, has been investigated. The behavior of a nonlinear interface between glass and isotropic MBBA is also described. It is known ${ }^{14}$ that IPs of nematics are nonlinear liquids of the Kerr type. Therefore, they are suitable to the situation described in Kaplan's works. In both situations, a predominance of the thermal effects has been observed. The anchoring forces have been found to inhibit the molecular reorientation near the boundary, not only in nematic, but also in isotropic phase.

\section{GLASS-NEMATIC INTERFACE UNDER TOTAL INTERNAL REFLECTION}

The experimental setup shown in Figure 1 has been used to study the glass nematic interface. A TE input beam impinges on the interface, characterized by a homeotropic alignment. In this case, whenever a part of the field is transmitted to the nematic layer, the molecules tend to orient along the electric field direction. Hence, an increase in the refractive index is obtained. However, if there is a strong anchoring of the molecules at the boundary, the refractive index can be only modified at a certain distance from the interface. If the evanescent field is able to produce reorientation at such distances, then switching would be produced between TIR and Frustrated Total 


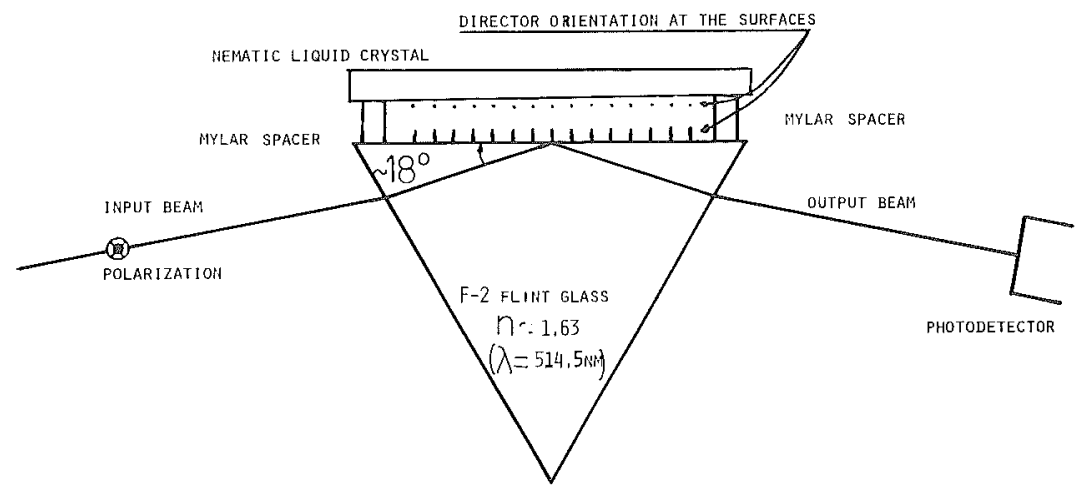

FIGURE 1 Experimental set-up.

Internal Reflection (FTIR). This switching has not been proved yet to display a hysteresis, and this is one of the unknowns of the present study.

In the previous work by Khoo, ${ }^{9}$ the experimental setup consisted of a homeotropically oriented nematic between two prisms. The extra prism had a high refractive index in order to collect the transmitted beam. Here, a homogeneous alignment has been used at the other boundary of the nematic film as indicated in Figure 1. The hybrid alignment provides an initial bend distortion which avoids the threshold effect when the field is normal to the director ${ }^{16}$. As can be seen from Figure 2, this bend also reduces the minimum depth necessary for the evanescent field to induce reorientation. Moreover, the anchoring of the boundary molecules causes the ordinary refractive index to control the optical properties (and hence the TIR condition to be met) at both boundaries of the nematic film if the homeotropic alignment (Figure 2b) is used. A double FTIR should be necessary in order to obtain a transmitted wave.

The main problem in these experiments is to see if the evanescent field can reorientate the molecules at some distance from the boundary. In order to illustrate this problem, let us make some calculations on the field intensity and penetration depth of the evanescent field. The Fresnel formula for TE polarization takes a complex value for the TIR case. Its module yields the following amplitude of the evanescent field at the boundary $(z=0)$ :

$$
E_{\mathrm{e}}=2 \sin \varphi / \sin \varphi_{c} E_{\mathrm{i}}
$$

where $E_{\mathrm{e}}$ and $E_{\mathrm{i}}$ are the evanescent and incident wave amplitudes; 
(a)
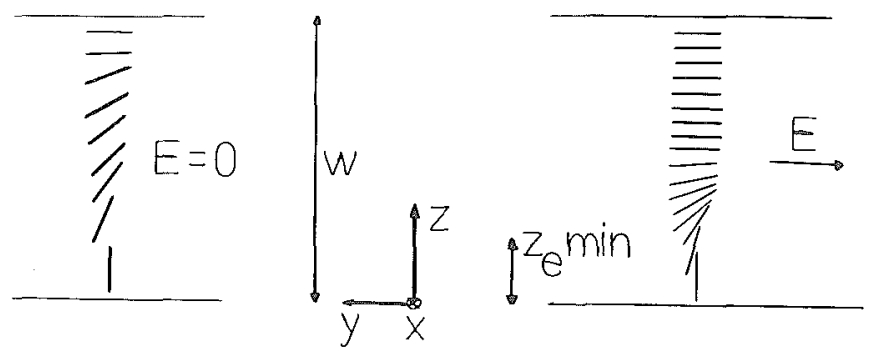

(b)
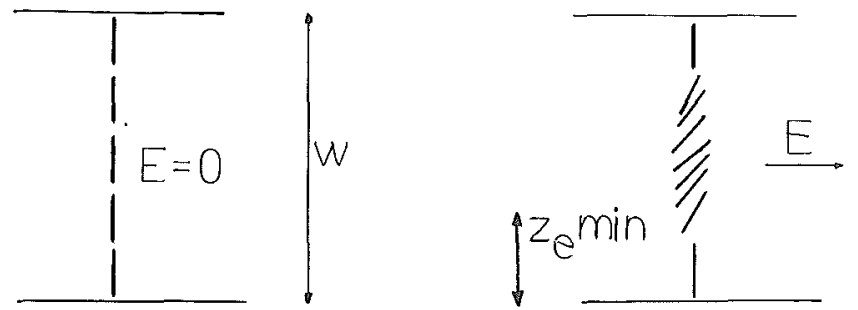

FIGURE 2 Orientation of the molecules at the nematic film without any field and with an ideal constant electric field: (a) hybrid aligned configuration; (b) homeotropic configuration.

$n_{\mathrm{t}}$ and $n_{\mathrm{i}}$, the refractive indices of the "transmission" and incidence media. $\varphi$ is defined as the angle between interface and wave normal. Near the critical angle $\varphi_{c}$,

$$
\varphi=\varphi_{c}-\Delta \varphi, \text { where } \Delta \varphi<<1
$$

and (1) becomes

$$
E_{\mathrm{e}} \approx 2\left(1-\Delta \varphi / \tan \varphi_{c}\right) E_{\mathrm{i}}
$$

The penetration depth defined in the usual way ${ }^{17}$ is

$$
z_{\mathrm{e}} \approx \frac{\lambda_{0} / 2 \pi n_{\mathrm{t}}}{\left(2 \Delta \varphi \tan \varphi_{c}\right)^{1 / 2}}
$$

In our case, the transmission medium is $\operatorname{MBBA}\left(n_{\mathrm{t}}=n_{0}=1,544\right)$ and the incidence medium an F-2 prism $\left(n_{\mathrm{i}}=1.627\right.$ at $\lambda=514.5$ $\mathrm{nm})$. Hence, the values for critical angle and penetration depth are

$$
\begin{aligned}
& \varphi_{c} \approx 18.38^{\circ} \\
& z_{\mathrm{e}} \approx 0.2213 \lambda_{0} /(\Delta \varphi)^{1 / 2}
\end{aligned}
$$


and

$$
E_{\mathrm{e}} \approx 2 E_{\mathrm{i}}(1-3 \Delta \varphi)
$$

The penetration depth is found to be about one wavelength $\left(z_{\mathrm{e}} \approx\right.$ $\left.\lambda_{0}\right)$ when $\Delta \varphi \approx 3^{\circ}$. In these conditions $E_{e}(\varphi) \approx 0.85 E_{e}(\varphi c)$ being $E_{\mathrm{e}}\left(\varphi_{\mathrm{c}}\right)=2 E_{\mathrm{i}}$. Therefore, the penetration depth of the evanescent field is a more critical parameter than its amplitude at the boundary. A small misalignment of the incidence angle can reduce the penetration depth to a few wavelengths.

The problem gets more complicated when the incident field is not a plane wave, but a Gaussian beam. In this case light impinges on the interface with different angles at the same time, and some of them can be quite far away from the critical one, especially if one wants the whole beam to be totally reflected. The energy impinging under those angles do not contribute substantially to the evanescent field. Several authors developed numerical solutions in order to analyze such transversal inhomogeneity. ${ }^{18}$ Even though they obtain contradictory results, they agree in the absence of $O B$. This fact seems to be confirmed by some experimental work. ${ }^{8}$

\section{EXPERIMENTAL RESULTS FOR THE GLASS-NEMATIC INTERFACE}

Several MBBA film thicknesses have been used with the configuration of Figure 1. Figure 3 a shows reflected intensity versus incident intensity for two cell thicknesses, $90 \mu \mathrm{m}$ and $20 \mu \mathrm{m}$. Thinner films display a higher nonlinearity for low intensities. The absence of switching points suggests that hysteresis and nonlinearity are mainly due to thermal effects. The stronger effect in thinner films can be due to the smaller volume of MBBA to be heated. There is also some absorption (and hence heat generation) in the prism; otherwise it would behave as a heat sink. In fact, very high laser powers can induce the damage to the glass. However, repetitive experimentation is very difficult because of the small lifetime of thin hybrid aligned cells. ${ }^{19}$ This time may be reduced when a strong laser beam is acting on them.

Nonlinear effects are also observed in thick cells at higher intensities as shown in Figure 3b. If the clearing temperature is reached, MBBA becomes isotropic, the anchorage of the molecules vanishes and the initial alignment is only recovered after several hours when the affected region is small. The incidence angle was around $18^{\circ}$, the 


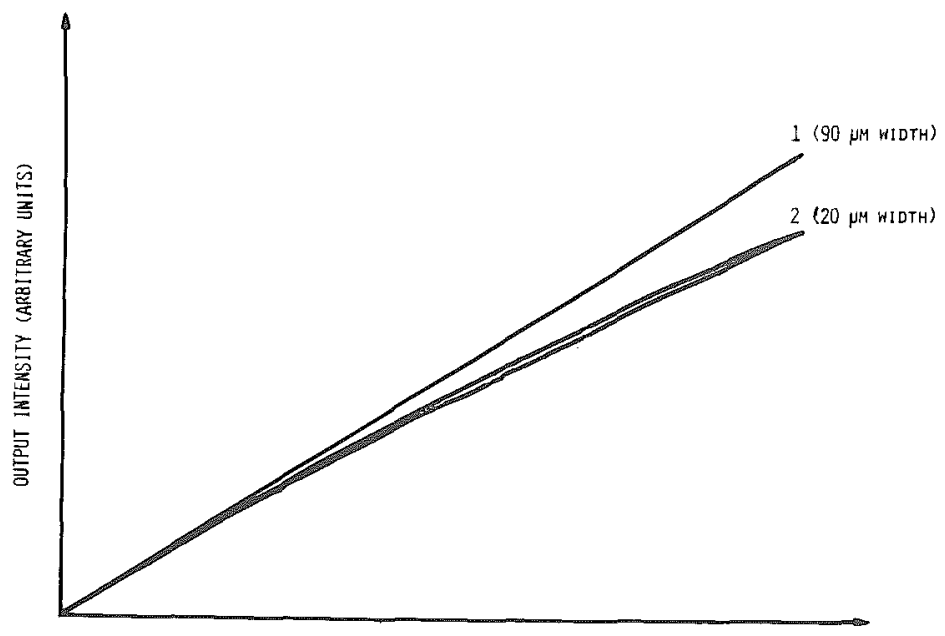

INPUT INTENSITY (ARBITRARY UNITS)

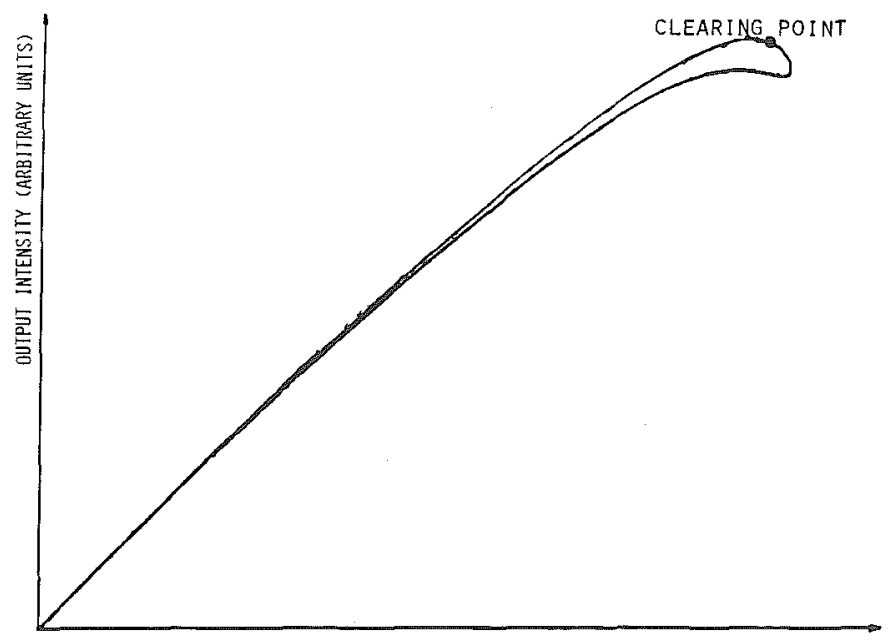

INPUT INTENSITY (ARBITRARY UNITS)

FIGURE 3 Reflected intensity versus incident intensity with initial TIR: (a) behavior at low intensities for two nematic film thicknesses; (b) behavior when the incident intensity increases until the liquid crystal clears to isotropic phase. 
critical angle was $18.4^{\circ}$, and the temperature was $25^{\circ} \mathrm{C}$. The scanning times for the results shown in Figure 3 were between 2 and 5 minutes. A more careful study shows that only points in the lower branch are stable. When thermal equilibrium is established this branch is reached.

When the incidence angle increases and a transmitted wave arises, the typical diffraction pattern produced by reoriented nematics appears in the transmitted wave. However, the reflected wave is not affected by reorientation and its behavior is dominated by the thermal effect. This fact shows that the reorientation is not produced at the interface, but near the central part of the film. This is consistent with the assumption of strong anchoring.

The setup geometry was modified in order to definitely elucidate if there is an interaction between the evanescent field and the reorientable region. This interaction may have not been detected in the previous experiments due to the masking of the thermal effect. The beam impinging on the interface was substituted by a low intensity $\mathrm{He}-\mathrm{Ne}$ laser $(5 \mathrm{~mW})$. An external normally incident high intensity $\mathrm{Ar}^{+}$laser beam was focused on the MBBA film through the glass plate opposite to the prism. In this way, the behavior of the low intensity laser, induced by the changes produced in the nonlinear medium can be tested. When both beam polarizations are parallel, the result is a sort of "reflection coefficient-versus-transmitted intensity" plot which can illuminate the preceding results. Figure 4 shows the result when a $110 \mu \mathrm{m}$ homeotropic MBBA film is used. The clearing point is reached and hysteresis effects arise for incidence intensities of about $37 \mathrm{~kW} / \mathrm{cm}^{2}$. This is due to a misalignment of the molecules induced by a quick cooling. The originally homeotropic alignment is not recovered until several hours. The study with a PCB homeotropic film does not show any dependence between reflected and control beams. PCB being a colorless nematic, the thermal effect is smaller, which suggests that the dependence shown in Figure 4 is only due to thermal index variation. It seems that laser heating induces a modification of the anchoring forces at the surface, and both reorientation and thermal indexing contribute to the rise of the refractive index. In fact, a nonlinear dependence with a slower response time has been observed. Figure 4 shows a metastable situation; indeed a sharper variation can be obtained after a long time. However, it can be concluded that molecular reorientation near the boundary cannot be obtained in the absence of thermal effect, and the interaction between the evanescent field and the reorientation can only be produced whenever anchoring is weak. 


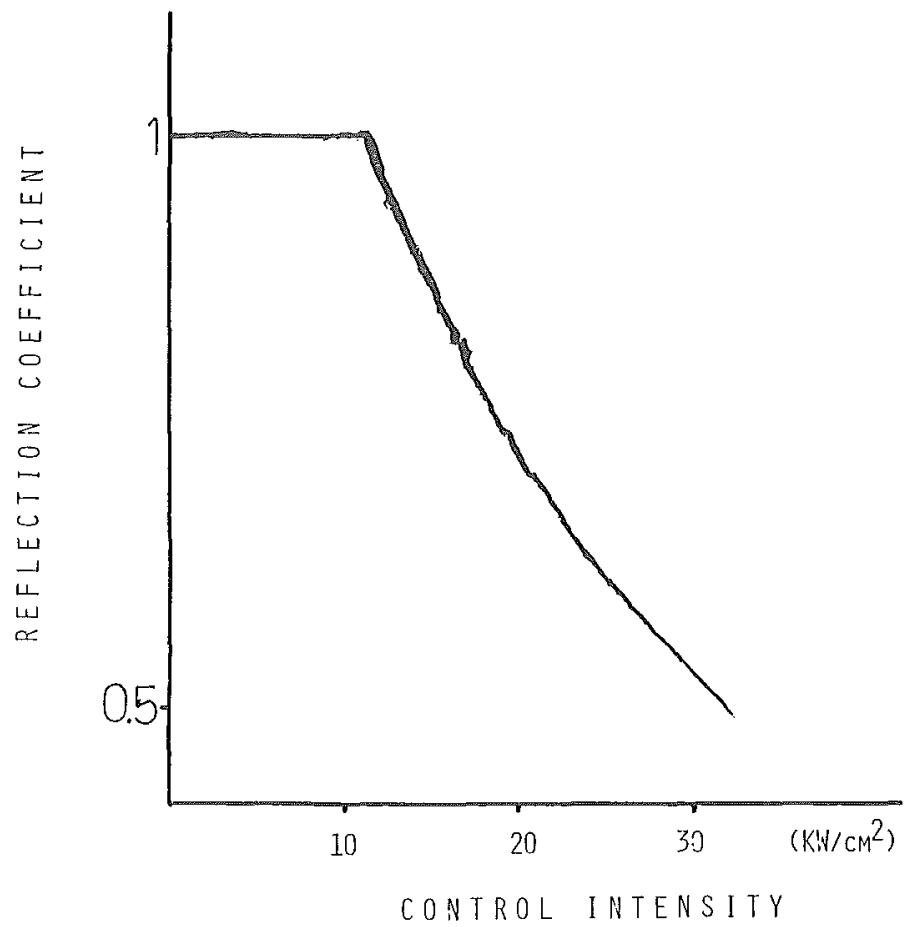

FIGURE 4 Behavior of the reflected beam as a function of the control beam intensity. The transmission medium is an homeotropic film of MBBA, and we are at TIR when control intensity is low.

\section{EXPERIMENTAL RESULTS FOR THE GLASS-ISOTROPIC MBBA INTERFACE}

Thermal variations of refractive indices in NP are strong, especially near the nematic-isotropic transition. ${ }^{20}$ On the other hand the variation in IP is very smooth. It should be expected that the isotropic MBBA would behave as a Kerr liquid with a negligible thermal dependence, and therefore, suitable for experimentation in nonlinear interfaces. In order to reach TIR, an SF-10 prism $(n \approx 1.74$ at 514.5 $\mathrm{nm}$ ) has been used as incidence medium, for refractive index of MBBA is about 1.64. The observed behavior has been linear: the system remains under TIR even for intensities high enough to produce laser damage in the glass.

When the incidence angle surpasses the critical one, the reflected beam behaves nonlinearly as shown in Figure 5. This beam keeps its 


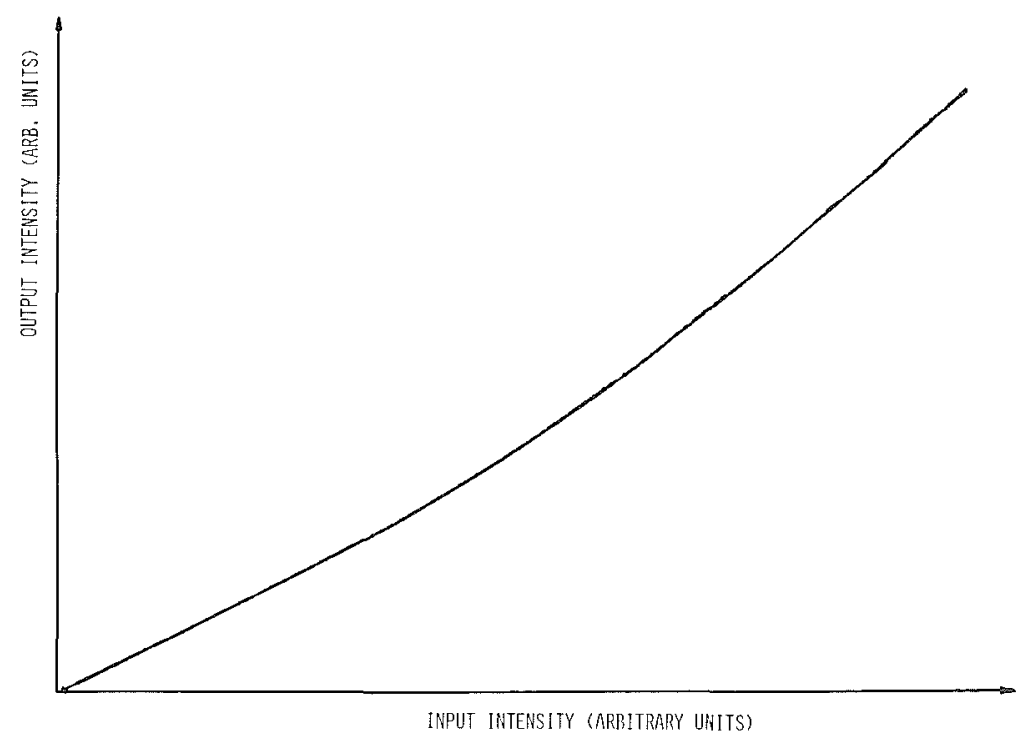

FIGURE 5 Reflected intensity versus incident intensity when incidence angle allows transmission. The incidence medium is an SF-10 prism, and the transmission one is MBBA in isotropic phase.

Gaussian shape, while the transmitted one shows a diffraction pattern due to transversal inhomogeneities in the refractive index. Figure 5 suggests that high intensities enhance the reflection and therefore, the refractive index of the nonlinear medium decreases.

The alternative setup (low power reflected beam and high power external control beam) was used to obtain a more complete information. Experiments show that when the red beam is under TIR, the reflection is not affected by the control beam. When the transmitted beam through the ( $40 \mu \mathrm{m}$ thick) nonlinear medium is present, the reflected intensity behaves as shown in Figure 6. An increase of the reflectivity is produced by the external high intensity beam confirming the negative nonlinearity suggested in Figure 5. This effect can also be proved by observing the transmitted red beam through a filter which absorbs the $\mathrm{Ar}^{+}$scattered light. As the control beam intensity increases, the transmitted low power beam becomes more and more grazing until TIR is met and it disappears.

This result is independent of the wavelength of the control beam. It can also be obtained with crossed beam polarizations. However, it is known that Kerr effect is based on enhanced molecular order (hence anisotropy) induced by the optical field. So we can speak of an extraordinary susceptibility (parallel to the field polarization) and 


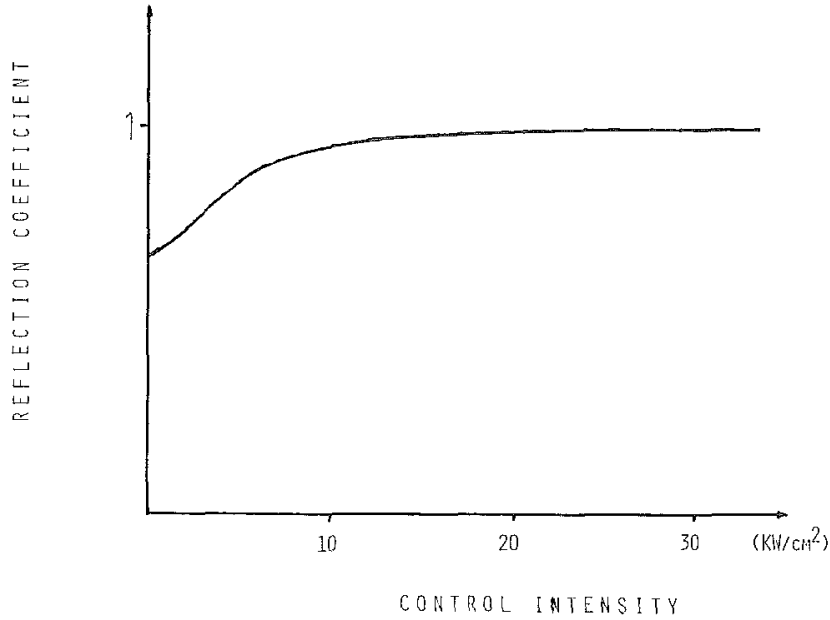

FIGURE 6 Behavior of the reflected beam as a function of the control beam intensity. The transmission medium is a film of MBBA in isotropic phase and the incidence angle allows an initial transmission with low control intensities.

an ordinary one (in any other of its orthogonal orientations). Their value should be ${ }^{14,21}$

$$
\begin{aligned}
& \chi_{e}=\bar{\chi}+2 / 3 \Delta \chi_{0} Q \\
& \chi_{o}=\bar{\chi}-1 / 3 \Delta \chi_{0} Q
\end{aligned}
$$

where $Q$ is the order parameter, proportional to $|E|^{2}$.

Our experiments, on the contrary, indicate that,

$$
\chi_{\mathrm{e}} \approx \chi_{0} \approx \bar{\chi}(\mathrm{T})
$$

where the temperature dependence has been included to explain the observed negative nonlinearity. As it has been indicated above, the refractive index in IP smoothly decreases with temperature.

The observed behavior shows that the term containing the molecular order parameter $Q$ is negligible. This can be justified through two different arguments.

The first of them comes from the relation between this parameter and the temperature ${ }^{14}$ :

$$
Q \propto\left|E_{0}\right|^{2} /\left(\mathrm{T}-\mathrm{T}^{*}\right)
$$

where $T^{*}$ is a temperature near-but below-the nematic-isotropic transition. As the temperature dependence of $\bar{\chi}(T)$ is very weak, an 
observable variation of the refractive index should have to be produced by a high temperature increase. But when the temperature is very high, the order parameter vanishes.

The second argument might be the difficulty in reorienting the molecules which are in contact with the surface. This means that there exist anchoring forces in the surfaces. This assumption is supported in the formation of a high aperture, annular-shaped diffraction pattern both in the transmitted and the control beams, but never in the reflected one. Thus, it can be concluded that a sharp transversal inhomogeneity of the refractive index is produced in the nonlinearmedium, but away from the boundary. Such inhomogeneity cannot be due to a thermal effect, because, in case of heat transmission the transversal variations of temperature should be smooth. In fact, thermal self-defocusing never produces such diffraction patterns, but a smooth broadening of the beam. ${ }^{5}$ In conclusion, a Kerr effect is present in the bulk whereas a thermal effect is dominant near the interface, showing a negative nonlinearity. Negative nonlinearities also play an important role in bistability at nonlinear interfaces, ${ }^{22}$ but they are not so useful when coming from a thermal heating. In this case, the incidence medium can heat the nonlinear one and modify its refractive index, even when no optical field is present in it.

\section{CONCLUSIONS}

The behavior of nematic liquid crystals as nonlinear medium in nonlinear interfaces has been studied. Their usefulness as materials which can exhibit a "positive" nonlinearity with applications in optical bistability has been analyzed. The results show that both in the nematic and isotropic phase there are anchoring forces at the boundary that inhibit the action of the evanescent field. The penetratioin depth of this surface wave is smaller than the one needed to reach the "reorientable" part of the nematic. On the other hand, a strong thermal nonlinear effect appears in a colored liquid crystal such as MBBA, and in much lower degree, in a transparent liquid crystal as PCB. This thermal index variation induced by laser heating displays a "positive" nonlinearity in homeotropic films of nematic, and a negative one in isotropic phase. The thermal variation of the refractive index, however, can be produced by another kind of heating different from the action of the laser light in the nematic. In particular, isotropic phases of nematic substances exhibit high refractive indices. So it is necessary to use a glass with a higher refractive index to achieve the TIR. Usually, glasses with high refractive indices are highly dispersive too. That means that there is an absorption band near the working 
wavelengths. Therefore, a laser induced glass heating can be expected, and this can produce a temperature rise in the nematic near the boundary, which is not directly produced by the presence of an optical field within it.

Summarizing, it seems that nematic liquid crystals are not suitable materials to be used in the study of optical nonlinear interfaces if control of the refractive index with the transmitted field is attempted.

\section{Acknowledgment}

This work has been supported by the Spanish Comision Asesora de Investigación Cientifica y Técnica, grant No. $1564 / 82$.

\section{References}

1. Optical Bistability, eds. C. M. Bowden, M. Ciftan and H. R. Robl (Plenum N.Y. 1981).

2. Optical Bistability 2, eds. C. M. Bowden, H. M. Gibbs, and S. L. McCall, (Plenum, N.Y. 1984).

3. Optical Bistability III, eds. by H. M. Gibbs, P. Mandel, N. Peyghambarian, and S. D. Smith (Springer-Verlag, Berlin, 1986).

4. H. M. Gibbs, Optical Bistability: Controlling Light with Light (Academic Press, Orlando, 1985).

5. Y. R. Shen, The Principles of Nonlinear Optics (Wiley \& Sons, New York 1984).

6. F. A. Hopf and G. I. Stegeman, Applied Classical Electrodynamics, Volume 2: Nonlinear Optics (Wiley \& Sons, New York 1986).

7. A. E. Kaplan, JETP, 24, 114-119 (1976); Sov. Phys. JETP, 45, 896-905 (1977).

8. P. W. Smith, J. P. Hermann, W. J. Tomlinson, and P. J. Maloney, Appl. Phys. Lett. 35, 846-848, (1979); P. W. Smith, W. J. Tomlinson, P. J. Maloney and J. P. Herman, IEEE J. Quant. Electron. QE-17, 340-348, (1981); P. W. Smith and W. J. Tomlinson, IEEE J. Quant. Electron. QE-20, 30-36 (1984).

9. I. C. Khoo, Appl. Phys. Lett. 40, 645-647 (1982).

10. J. A. Martín Pereda, XIII International Quantum Electronics Conference, IQEC '84, WII 13, Anaheim (CA), June 18-21, 1984; SPIE 492, 370 (1984).

11. H. Vach, C. T. Seaton, G. I. Stegeman, and I. C. Khoo, Opt. Lett. 9, 238-240 (1984).

12. E. S. Goldburt and P. St. J. Russell, Appl. Phys. Lett. 46, 338-340 (1985).

13. Y. R. Shen, Phil. Trans. R. Soc. Lond. A 313, 327-332 (1984); see also, Ref. 3, pp. $148-151$.

14. I. C. Khoo and Y. R. Shen, Opt. Eng. 24, 579-585 (1985).

15. I. C. Khoo and J. Y. Hou, J. Opt. Soc. Am. B2, 761-766 (1985).

16. G. Barbero, F. Simoni, and P. Aiello, J. Appl. Phys. 55, 304-311 (1984).

17. See, for example, K. Shimoda, "Introduction to Laser Physics," (Springer-Verlag, Berlin) p. 43 (1984).

18. D. Marcuse, Appl. Opt. 19, 3130-3139 (1980); W. J. Tomlinson, J. P. Gordon, P. W. Smith, and A. E. Kaplan, Appl. Opt. 21, 2041-2051 (1981).

19. J. F. Palierne, N. V. Madhusudana, G. Barbero, and G. Durand, 10th International Liquid Crystal Conference, York (U.K.), 15-21 July, 1984.

20. I. C. Khoo and R. Normandin, IEEE. J. Quant. Electron., QE-21, 329-335 (1985).

21. G. K. L. Wong and Y. R. Shen, Phys. Rev. A10, 1277-1284 (1974).

22. A. E. Kaplan, IEEE J. Quant. Electron. QE-17, 336-340 (1981). 